\title{
ÉLIMINATION DES QUANTIFICATEURS DANS LES PAIRES DE CORPS ALGÉBRIQUEMENT CLOS
}

\author{
FRANÇOISE DELON \\ Équipe de Logique Mathématique, IMJ, \\ CNRS-Université Paris 7-UPD, France \\ Received 7 February 2012 \\ Revised 13 July 2012 \\ Accepted 13 July 2012 \\ Published 27 September 2012
}

\begin{abstract}
Axiomatisation d'une structure et description de ses ensembles définissables sont deux éléments de description essentiels offerts par la théorie des modèles. Un résultat d'élimination des quantificateurs produit une description concrète des ensembles définissables. Nous montrons ici que les paires de corps algébriquement clos éliminent les quantificateurs dans le langage des anneaux enrichi des prédicats de disjonction linéaire au-dessus du petit corps et des fonctions donnant les composantes linéaires de la première variable sur les suivantes. Les paires denses de corps valués algébriquement clos éliminent les quantificateurs dans le langage précédent enrichi du prédicat div.
\end{abstract}

Keywords: Quantifier elimination; model theory of fields; pairs of fields; algebraically closed fields; algebraically closed valued fields; ACVF.

AMS Subject Classification: 03C10, 03C60, 12J10, 12L12

\section{Introduction}

La théorie des modèles a plusieurs moyens de décrire une structure. Elle peut par exemple l'axiomatiser. On obtient ainsi des théorèmes de transfert, dont le plus célèbre est sans doute la résolution par $\mathrm{Ax}$ et Kochen de la conjecture d'Artin: à $d$ fixé, pour presque tout entier premier $p$, le corps $\mathbb{Q}_{p}$ est $C_{2}(d)$; le transfert se fait ici d'un ultraproduit des corps $\mathbb{F}_{p}((X))$, qui est $C_{2}$ parce que les corps finis sont $C_{1}$, vers le même ultraproduit des $\mathbb{Q}_{p}$. Elle peut aussi décrire les ensembles définissables. Les résultats d'élimination des quantificateurs entrent dans cette catégorie. Les deux opérations sont en principe indépendantes l'une de l'autre. Ainsi on ne sait pas axiomatiser le corps exponentiel des réels (sauf à admettre la conjecture de Schanuel), mais on sait qu'il est modèle-complet, ce qui signifie que tout ensemble définissable est combinaison booléenne d'ensembles définissables par les seules formules existentielles. On sait aussi et surtout qu'il est o-minimal, ce qui implique en particulier que tout sous-ensemble définissable (d'une puissance de $\mathbb{R}$ ) a un nombre fini de composantes connexes, qui se trouvent de plus être 
définissables. Néanmoins les deux vont souvent de pair, les corps algébriquement clos représentant le paradigme de la structure dont les propriétés modèle-théoriques sont totalement explicitées : le fait d'être algébriquement clos s'exprime au 1er ordre, la caractéristique également, et les deux ensemble axiomatisent complètement le corps. De plus, ces corps éliminent les quantificateurs dans le langage des anneaux, ce qui est une autre façon de dire que la classe des constructibles est stable par projection.

Nous considérons dans cet article des paires de corps algébriquement clos. Une extension, ou paire, de corps $K \subseteq L$ va être lue comme une structure de base $L$ qui, en plus de la structure de corps de $L$, comporte un prédicat unaire décrivant l'appartenance à $K$. Il apparaît qu'une paire de corps algébriquement clos est complètement axiomatisée par les axiomes exprimant que les deux corps sont algébriquement clos, d'une caractéristique donnée, et que l'inclusion est propre. C'est Tarski le premier qui a considéré ce genre de question, lorsqu'il s'est interrogé sur la décidabilité de la paire $\left(\mathbb{R}, \mathbb{Q}^{r}\right)$ constituée du corps des réels et de la clôture réelle des rationnels. Abraham Robinson a répondu par l'affirmative, dans le même article où il a axiomatisé les paires de corps algébriquement clos. Pour axiomatiser $\left(\mathbb{R}, \mathbb{Q}^{r}\right)$, il suffit de dire que les deux corps sont réels clos, que l'inclusion est propre, et que le petit corps est dense dans le grand. La théorie des paires de structures a plus récemment trouvé d'importantes connexions avec la théorie de la stabilité. On peut caractériser une structure stable, disons $M$, par le fait que la trace sur $M^{n}$ d'une partie de $N^{n}$ définissable dans une extension élémentaire arbitraire $N$ de $M$ est définissable dans $M$ elle-même, en exigeant de plus que cela reste vrai pour toute structure satisfaisant les mêmes axiomes que $M$. C'est évidemment le cas d'un corps algébriquement clos: la trace sur $K^{n}$ d'un fermé de Zariski de $L^{n}$ est un fermé de Zariski de $K$; c'est évidemment faux pour un corps réel clos qui n'est pas $\mathbb{R}$ : par exemple la trace sur $\mathbb{Q}^{r}$ du segment ]- $-\pi[$ n'est certainement pas définissable dans $\mathbb{Q}^{r}$. Nous donnons ici un résultat d'élimination des quantificateurs qui permet de traiter ce genre de questions. Ce résultat n'est en soi guère surprenant et n'apporte rien de bien neuf dans le cas des paires de purs corps algébriquement clos, mais c'est un langage explicite, dont la simplicité et le contenu algébrique lui permettent de s'adapter à de nombreuses paires de corps ou expansions de paires de corps. Nous présentons ici le cas des paires denses de corps valués algébriquement clos, qui a des applications en $C$-minimalité.

Décrivons plus précisément nos résultats. On considère l'extension de corps $K \subseteq$ $L$ comme une structure $\mathbb{L}$ de base $L$ et de langage $\{0,1,+,-, \cdot, E\}$, où $E$ est un prédicat unaire ayant l'interprétation $E(x)$ ssi $x \in K$. Comme nous l'avons dit, dans le cas où $K$ et $L$ sont algébriquement clos et où l'extension est propre, ces propriétés, plus la caractéristique de $K$ et $L$, axiomatisent complètement $\mathbb{L}$. Cela est connu depuis l'article [12] d'Abraham Robinson, où il donne également un langage dans lequel la structure est modèle-complète. Gérard Leloup a ensuite décrit un langage permettant l'élimination des quantificateurs [7]. Nous proposons un autre 
langage d'élimination, qui, contrairement à celui de Leloup, ajoute de nouvelles fonctions, et change donc la notion de sous-structure. ${ }^{a}$

Enrichissons le langage d'une famille de symboles de prédicats $l_{n}$, pour $n \in \mathbb{N} \geq 2$, où chaque $l_{n}$ est d'arité $n$, et d'une famille de symboles de fonctions $f_{n, i}$ dárité $n+1$, pour $n \in \mathbb{N}^{\geq 2}$ et $1 \leq i \leq n$, tous définissables dans $\mathbb{L}$. La relation $l_{n}$ est définie comme suit :

$l_{n}\left(x_{1}, \ldots, x_{n}\right)$ ssi $x_{1}, \ldots, x_{n}$ sont linéairement indépendants au-dessus de $E$.

Ainsi " $l_{n}\left(x_{1}, \ldots, x_{n}\right) \wedge \neg l_{n+1}\left(x_{1}, \ldots, x_{n}, y\right)$ " exprime que $y$ appartient au $E$-espace vectoriel librement engendré par les $x_{1}, \ldots, x_{n} ; y$ se décompose alors sur cette base, et les fonctions $\left(f_{n, i}\right)_{1 \leq i \leq n}$ donnent les composantes:

$$
\begin{gathered}
z=f_{n, i}\left(y, x_{1}, \ldots, x_{n}\right) \leftrightarrow \\
\left\{l_{n}\left(x_{1}, \ldots, x_{n}\right) \wedge \exists\left(z_{j}\right)_{j=1}^{n}\left[z=z_{i} \wedge y=\sum_{j=1}^{n} z_{j} x_{j} \wedge \bigwedge_{j=1}^{n} E\left(z_{j}\right)\right]\right\} .
\end{gathered}
$$

Théorème 1. Les paires propres de corps algébriquement clos éliminent les quantificateurs dans le langage $\mathcal{L}^{f}:=\left\{0,1,+,-, \cdot,\left(l_{n}\right)_{n \in \mathbb{N} \geq 2},\left(f_{n, i}\right)_{n \in \mathbb{N} \geq 2,1 \leq i \leq n}\right\}$.

Théorème 2. Les paires propres et denses de corps valués algébriquement clos éliminent les quantificateurs dans le langage $\mathcal{L}^{f} \cup\{\mid\}$, où $\mid$ est le prédicat binaire $x \mid y$ ssi $v(x) \leq v(y)$.

Corollaire 3. Soit $\mathbb{M}$ une paire de corps algébriquement clos et un sous-corps $K \subseteq M$.

(1) Alors la clôture algébrique au sens de la théorie des modèles de $K$ dans $\mathbb{M}$ est la clôture algébrique au sens de la théorie des corps $C^{a}$ de $C=K\left(f_{n, i}(K) ; n \in\right.$ $\left.\mathbb{N}^{\geq 2}, 1 \leq i \leq n\right)$, et sa clôture définissable est la clôture parfaite $C^{p^{-\infty}}$.

(2) Si de plus $M$ porte une valuation faisant de ( $\mathbb{M}, v)$ une paire dense, alors la clôture algébrique de $K$ dans $(\mathbb{M}, v)$ reste $C^{a}$ et sa clôture définissable y est la clôture henselienne de $C^{p^{-\infty}}$.

\section{Le cas des purs corps algébriquement clos}

\subsection{Outils algébriques}

Un outil essentiel pour étudier les paires de corps est la disjonction linéaire. Nous en rappelons la définition ainsi que quelques résultats classiques, qu'on trouve par exemple dans [5], III, 1 (où nous traduisons free par algébriquement disjoints).

Définition 4. Deux extensions $K$ et $L$ d'un corps $C$, contenues dans un même surcorps, sont linéairement (resp. algébriquement) disjointes au-dessus de $C$ lorsque

all existe également un langage d'élimination provenant de l'étude des belles paires par Bruno Poizat dans [9]. 
des éléments de $K$ qui sont linéairement (resp. algébriquement) indépendants audessus de $C$ le restent au-dessus de $L$.

Une extension de corps $K \subseteq L$ est dite régulière lorsque $L$ et $K^{a}$ sont linéairement disjoints au-dessus de $K$.

Fait 5. Les corps $K$ et $L$ sont linéairement disjoints au-dessus de $C$ ssi l'anneau qu'ils engendrent est canoniquement isomorphe à $K \otimes_{C} L$. Ils sont algébriquement disjoints au-dessus de $C$ ssi le degré de transendance au-dessus de $C$ du corps qu'ils engendrent est la somme de leurs degrés de transcendance. Ces deux notions d'indépendance sont donc symétriques en $K$ et $L$.

Notations. Notons $K l d_{C} L$ pour exprimer que deux corps $K$ et $L$ (ayant une extension commune) sont linéairement disjoints au-dessus d'un sous-corps commun $C, K a d_{C} L$ pour exprimer qu'ils sont algébriquement disjoints ; $K L$ désigne le corps qu'ils engendrent.

Fait 6. (1) $K l d_{C} L$ implique $K \cap L=C$.

(2) Transitivité et réciproque: si $M$ est une extension de $L$, alors $K l d_{C} M$ équivaut à : $K l d_{C} L$ et $L K l d_{L} M$.

(3) Si l'extension $C \subseteq K$ est algébrique et l'extension $C \subseteq L$ régulière, alors $K l d_{C} L$.

(4) Si $K l d_{C} L$ et si l'extension $C \subseteq K$ est régulière, alors l'extension $L \subseteq L K$ est régulière.

Fait 7. (1) $K l d_{C} L$ implique $K a d_{C} L$.

(2) Si $C \subseteq K$ est une extension régulière, alors $K a d_{C} L$ implique $K l d_{C} L$. En particulier si $C$ est un corps algébriquement clos, $K a d_{C} L$ implique $K l d_{C} L$.

\subsection{Le langage $\mathcal{L}^{c, f}:=\mathcal{L}^{f} \cup\left\{{ }^{-1}\right\}$}

Notations. Désormais une paire de corps sera désignée par une notation du type $\mathbb{M}=\left(M, E_{M}\right)$. Si $\mathbb{M}$ est une paire de corps et $\mathrm{A}$ un sous-corps de $M, E_{A}$ désigne $A \cap E_{M}$ et $\mathbb{A}$ la paire $\left(A, E_{A}\right)$.

Remarquons que $E$ et $l_{1}$ sont définissables sans quantificateurs dans $\mathcal{L}^{f}: E(x) \leftrightarrow$ $\neg l_{2}(1, x)$, et $l_{1}(x) \leftrightarrow x \neq 0$.

Fait 8. Etant donnés une paire de corps $\mathbb{M}$ et $A \subseteq M$, la paire $\left(A, A \cap E_{M}\right)$ est une $\mathcal{L}^{c, f}$-sous-structure de $\mathbb{M}$ ssi $A$ est un sous-corps de $M$ linéairement disjoint de $E_{M}$ au-dessus de $E_{A}$.

Corollaire 9. Soit une paire de corps $\mathbb{M}$, des sous-corps $K$ et $L$ de $M$, isomorphes par un isomorphisme envoyant $E_{K}$ sur $E_{L}$ et tels que $K l d_{E_{K}} E_{M}$ et $L l d_{E_{L}} E_{M}$. Alors $\left(K, E_{K}\right)$ et $\left(L, E_{L}\right)$ sont des $\mathcal{L}^{c, f}$-sous-structures isomorphes de $\mathbb{M}$. 
Corollaire 10. Soit $\mathbb{K} \subseteq \subseteq_{\mathcal{L}^{c, f}} \mathbb{M}$ et $X \subseteq M$ algébriquement libre sur $K E_{M}$. Alors $\left(K(X), E_{K}\right) \subseteq \mathcal{L}^{c, f} \mathbb{M}$.

Démonstration. Par hypothèse $K(X) a d_{K} K E_{M}$; cela implique $K(X) l d_{K} K E_{M}$ par le fait $7(2)$ puisque l'extension $K \subseteq K(X)$ est régulière, puis $K(X) l d_{E_{K}} E_{M}$ par transitivité (c'est-à-dire le fait $6(2))$.

Lemme 11. Etant données une paire de corps $\mathbb{M}$ et une $\mathcal{L}^{c, f}$-sous-structure $\mathbb{A} \subseteq$ $\mathbb{M}$, l'adjonction à $\mathbb{A}$ d'éléments de $E$ produit une $\mathcal{L}^{c, f}$-sous-structure de $\mathbb{M}$ dont le type d'isomorphisme est bien défini, c'est-à-dire: pour tout corps $B$ vérifiant $E_{A} \subseteq B \subseteq E_{M}$, la paire $\mathbb{A} B:=(A B, B)$ est une $\mathcal{L}^{c, f}$-sous-structure de $\mathbb{M}$ dont le type d'isomorphisme au-dessus de $\mathbb{A}$ ne dépend que du type d'isomorphisme du corps $B$ au-dessus de $E_{A}$.

Démonstration. Par disjonction linéaire et par le fait 5, le type d'isomorphisme du corps $A B$ ne dépend que de ceux de $A$ et de $B$ au-dessus de $E_{A}$. Et $A l d_{E_{A}} E_{M}$ implique $A B l d_{B} E_{M}$.

\subsection{La preuve de l'élimination des quantificateurs}

Fait 12. Si $\mathbb{M}$ est une paire de corps algébriquement clos et $\mathbb{A} \subseteq \mathbb{M}$ une $\mathcal{L}^{c, f}$-sousstructure, alors l'extension $E_{A} \subseteq A$ est régulière.

Démonstration. $E_{M}$ contient la clôture algébrique de $E_{A}$, qui est donc $l d_{E_{A}} A$.

Lemme 13. Etant données une paire de corps algébriquement clos $\mathbb{M}$ et une $\mathcal{L}^{c, f}$ sous-structure $\mathbb{A} \subseteq \mathbb{M}$, la paire $\mathbb{A}^{a}:=\left(A^{a}, E_{A}^{a}\right)$ est une $\mathcal{L}^{c, f}$-sous-structure de $\mathbb{M}$.

Démonstration. Par le lemme 11, $\mathbb{A} \subseteq_{\mathcal{L}^{c, f}}\left(A E_{A}^{a}, E_{A}^{a}\right) \subseteq_{\mathcal{L}^{c, f}} \mathbb{M}$. Ensuite, $E_{A}^{a}$ étant algébriquement clos, l'extension $E_{A}^{a} \subseteq E_{M}$ est régulière, donc aussi l'extension $A E_{A}^{a} \subseteq A E_{M}$ par le fait 6(4). Cela implique $A^{a} l d_{A E_{A}^{a}} A E_{M}$ par le fait 6(3), et enfin $A^{a} l d_{E_{A}^{a}} E_{M}$ par transitivité.

Proposition 14. Les paires propres de corps algébriquement clos éliminent les quantificateurs dans le langage $\mathcal{L}^{c, f}$.

Démonstration. Nous allons montrer l'élimination des quantificateurs en utilisant le critère de Shoenfield [13], que nous décrivons maintenant. On se donne $\mathbb{A}$ et $\mathbb{A}^{\prime}$, $\mathcal{L}^{c, f}$-sous-structures respectives des paires propres de corps algébriquement clos $\mathbb{M}$ et $\mathbb{N}$. On suppose $\mathbb{M}$ dénombrable, $\mathbb{N} \aleph_{1}$-saturé, et $\mathbb{A}$ et $\mathbb{A}^{\prime}$ isomorphes par un $\mathcal{L}^{c, f}$-isomorphisme $\sigma$. Il faut pouvoir plonger $\mathbb{M}$ dans $\mathbb{N}$ en prolongeant $\sigma$. On reproduit d'abord $E_{M}$ en $B \subseteq E_{N}$, isomorphe à $E_{M}$ par un isomorphisme (de corps) prolongeant $\sigma \uparrow E_{A}$; c'est possible parce que les corps algébriquement clos éliminent les quantificateurs dans le langage des corps. Par les lemmes 11 et $13, \mathbb{A}^{\prime} B$ est $\mathcal{L}^{c, f}$ isomorphe à $\mathbb{A} E_{M}$, et $\left(\mathbb{A}^{\prime} B\right)^{a}=: \mathbb{K}^{\prime}$ à $\left(\mathbb{A} E_{M}\right)^{a}=: \mathbb{K}$. On prend ensuite une base de transcendance $\left(t_{i}\right)_{i \in \alpha}$ de $M$ sur $K$ (où nécessairement $\alpha \leq \omega$ ). Par $\aleph_{1}$-saturation de $\mathbb{N}$, il existe des $\left(t_{i}^{\prime}\right)_{i \in \alpha}$ dans $N$, algébriquement indépendants sur $K^{\prime} E_{N}$. Par le 
fait $7(2), K^{\prime}\left(t_{i}^{\prime} ; i \in \omega\right)^{a}=: M^{\prime}$ est linéairement disjoint de $E_{N}$ sur $B$, donc $\left(M^{\prime}, B\right)$ et $\mathbb{M}$ sont $\mathcal{L}^{c, f}$-isomorphes par les corollaires 10 et 9 .

Démonstration du théorème 1. Les formules ci-dessous sont vraies dans toute paire de corps, pour tous $x_{1}, \ldots, x_{n}, y_{1}, \ldots, y_{n}, u, v$ :

$$
\begin{aligned}
\prod_{i=1}^{n} y_{i} & \neq 0 \rightarrow\left[l_{n}\left(\ldots, x_{i} y_{i}^{-1}, \ldots\right) \leftrightarrow l_{n}\left(\ldots, x_{i} \prod_{j=1, j \neq i}^{n} y_{j}, \ldots\right)\right], \\
v \prod_{i=1}^{n} y_{i} \neq 0 \rightarrow\left[f_{n, k}\left(u v^{-1}, \ldots, x_{i} y_{i}^{-1}, \ldots\right)\right. & \left.=f_{n, k}\left(u \prod y_{i}, \ldots, v x_{i} \prod_{j=1, j \neq i}^{n} y_{j}, \ldots\right)\right] .
\end{aligned}
$$

Cela permet d'éliminer le symbole ${ }^{-1}$ des termes apparaissant comme argument d'un prédicat $l_{n}$ ou d'une fonction $f_{n, i}$. Les atomes restants de $\mathcal{L}^{c, f}$ expriment la nullité de fractions rationnelles en $x_{1}, \ldots, x_{n}$ et $f_{n, k}\left(y, x_{1}, \ldots, x_{n}\right) ;^{-1}$ est clairement éliminable de telles formules.

Corollaire 15. La théorie des paires propres de corps algébriquement clos est modèle-complète dans le langage $\mathcal{L}^{l d}:=\left\{0,1,+,-, \cdot,\left(l_{n}\right)_{n \in \mathbb{N} \geq 2}\right\}$.

Remarque. Si $E(x)$ et $x \neq 0$, alors $f_{1,1}(1, x)=x^{-1}$.

Démonstration du corollaire 3, premier item. La clôture algébrique de $K$ dans $M$ contient clairement $C^{a}$. Réciproquement, ou bien $C^{a}$ est un modèle donc algébriquement clos, ou bien $K \subseteq E_{M}$ et dans ce cas $C=K$. Or tous les points de $M \backslash E_{M}$ ont même type sur $E_{M}$. Ainsi $K^{a}$ est algébriquement clos dans $E_{M}$ qui est algébriquement clos dans $M$. La clôture définissable de $K$ dans $M$ contient quant à elle $C^{p^{-\infty}}$ et réciproquement tout point de $C^{a} \backslash C^{p^{-\infty}}$ a un conjugué non trivial par un $C$-automorphisme du corps $C^{a}$; un tel automorphisme conserve globalement $E_{C}^{a}$ et est donc un $\mathcal{L}^{f}$-automorphisme.

\section{Les paires denses de corps valués algébriquement clos}

\subsection{Rappels sur les corps valués et les paires de corps valués}

Il est classique que les corps algébriquement clos non trivialement valués $(C V A C)$ éliminent les quantificateurs dans le langage $\mathcal{L}_{v}:=\left\{0,1,+,-, \cdot{ }^{-1}, A_{v}\right\}$ ou $\mathcal{L}_{\text {div }}:=$ $\{0,1,+,-, \cdot, \mid\}$, où $x \mid y$ signifie $v(x) \leq v(y)$ (la preuve se trouve implicitement chez Abraham Robinson [11] III 3.4 et 3.5). Contrairement à ce qui se passe pour les purs corps algébriquement clos, étant donnée une théorie complète $T$ de CVAC, la théorie des paires propres de modèles de $T$ est incomplète et indécidable [2]. Plusieurs complétions sont connues: les paires denses, les paires séparées, et certaines combinaisons des deux (d'après Leloup [6], après des travaux de Baur [1] qui s'était lui-même inspiré de Macintyre [8]). Le théorème 1 s'adapte bien à ces théories. Nous traitons ici le cas des paires denses qui ne requiert aucun symbole supplémentaire. 
Notation. Une paire de corps valués $(K, v) \subseteq(L, v)$ sera notée $(L, K, v)$, ou simplement $(L, K)$ s'il n'y a pas ambiguïté. Le groupe $v K^{x}$ sera noté simplement $v K$.

Définition 16. On appelle dense une paire de corps valués dans laquelle le petit corps est dense dans le grand pour la topologie de la valuation.

La densité s'exprime au premier ordre: la paire $(L, K, v)$ est dense ssi elle satisfait

$$
\forall x, \forall u \neq 0, \exists y, \quad E(y) \wedge v(x-y)>v(u) .
$$

Nous allons montrer que les paires strictes et denses de CVAC éliminent les quantificateurs dans le langage $\mathcal{L}_{\text {div }}^{f}:=\mathcal{L}^{f} \cup \mathcal{L}_{\text {div }}$. Nous rappelons d'abord quelques résultats de théorie des valuations nécessaires à la preuve.

Fait 17. Une valuation sur un corps admet toujours un prolongement à sa clôture algébrique. Les différents prolongements sont conjugués.

Démonstration. Voir par exemple [10], théorème 1, p. 166.

Fait 18. Soit $(L, K, v)$ une paire de corps valués, et $x, y \in L$ vérifiant $v(x), v(y)>$ $v K$. Alors $K(x)$ et $K(y)$ sont des corps valués isomorphes au-dessus de $K$.

Définition 19. Dans un corps valué $(K, v)$, on dira qu'une suite $\left(a_{\alpha}\right)_{\alpha<\alpha_{0}}$ indexée par des ordinaux est de Cauchy si $\alpha_{0}$ est limite et si, pour tout $\xi \in v K$, il existe $\alpha_{1}<\alpha_{0}$ tel que, pour tous $\alpha, \beta$ et $\gamma$ vérifiant $\alpha_{1}<\alpha<\beta<\gamma<\alpha_{0}$, on a $v\left(a_{\gamma}-a_{\beta}\right)>v\left(a_{\alpha}-a_{\beta}\right)>\xi$. On appelle limite de cette suite tout point $x$ vérifiant $v\left(x-x_{\alpha}\right)=v\left(x_{\beta}-x_{\alpha}\right)$ pour tous $\alpha$ et $\beta$ assez grands vérifiant $\alpha<\beta<\alpha_{0}$.

Il est à noter qu'une suite de Cauchy de $K$ ne reste pas de Cauchy dans une extension $L$ de $K$ lorsque $v K$ n'est pas cofinale dans $v L$. Elle y est seulement pseudoCauchy (pseudo-convergente selon la définition donnée par Kaplansky dans [4]). En particulier la suite peut alors admettre plusieurs limites dans $L$ : si $l$ en est une limite, tout point $x$ vérifiant $v(x-l)>v K$ est également limite. Dès lors qu'on ne s'intéresse qu'à leurs éventuelles limites, les suites de Cauchy indexées par la cofinalité du groupe $v K$ sont suffisantes. Le fait suivant se déduit des propriétés classiques des suites pseudo-Cauchy.

Fait 20. Une paire de corps valués $(L, K, v)$ est dense ssi tout point de $L \backslash K$ est limite d'une suite de Cauchy de $K$ sans limite dans $K{ }^{\text {b }}$ Si $K$ est algébriquement

\footnotetext{
${ }^{\mathrm{b}}$ Ce qui est également équivalent à ce que tout point de $L$ soit limite d'une suite de Cauchy de $K$. La situation est complètement différente pour les suites pseudo-Cauchy : étant donnée une extension arbitraire $(L, K, v)$ avec un groupe de valuation $v K$ qui ne soit pas $\mathbb{Z}$, un point $l \in L$ est toujours limite d'une suite pseudo-Cauchy de $K$ dès que $v(l) \geq v(k)$ pour un $k \in K$; parce que $v K \neq \mathbb{Z}$ il existe en effet dans $v K$ une coupure $<v(k)$ et donc une suite $\left(a_{\alpha}\right)_{\alpha<\alpha_{0}}$ de $K$ de valuation strictement croissante et $<v(k)$; cette suite est alors pseudo-Cauchy, et $0, k$ et $l$ en sont des limites.
} 
clos et si $x \in L$ est limite d'une suite de Cauchy $s$ de $K$ sans limite dans $K$, alors $s$ détermine complètement le type d'isomorphisme du corps valué $K(x)$ au-dessus de $K$.

Lemme 21. Soient donnés une paire dense de corps valués algébriquement clos $(L, K, v)$, un cardinal $i_{0}$, pour les ordinaux $i<i_{0}$ des éléments $a_{i}$ de $L$ algébriquement indépendants sur $K$, et $s_{i}=\left(a_{i, \alpha}\right)_{\alpha<\alpha_{0}}$ une suite de Cauchy de $K$ tendant vers $a_{i}\left(\alpha_{0}\right.$ est la cofinalité de $\left.v K\right)$. Alors les suites $s_{i}$ sont algébriquement indépendantes sur $K$ au sens suivant: pour tout $P \in K\left[X_{1}, \ldots, X_{n}\right] \backslash\{0\}$, pour tous ordinaux $i_{1}, \ldots, i_{n}<i_{0}$ tous distincts, il existe $\eta \in v K$ et un ordinal $\beta<\alpha_{0}$, tels qu'on ait, pour tous $\gamma_{1}, \ldots, \gamma_{n}$ avec $\beta<\gamma_{j}<\alpha_{0}, v\left(P\left(a_{i_{1}, \gamma_{1}}, \ldots, a_{i_{n}, \gamma_{n}}\right)\right)=\eta$. En conséquence les suites $s_{i}$ déterminent complètement le type d'isomorphisme audessus de $K$ du corps valué $K\left(a_{i} ; i<i_{0}\right)$.

Démonstration. Soit $(M, v)$ une extension de $(K, v)$ contenant, pour chaque $i<$ $i_{0}$, une limite $b_{i}$ de $s_{i}, M_{i}:=K\left(b_{j} ; j<i\right)^{a}, L_{i}:=K\left(a_{j} ; j<i\right)^{a}$. Alors $M_{i_{0}}$ et $L_{i_{0}}$ sont des corps valués isomorphes au-dessus de $K$, par un isomorphisme envoyant chaque $b_{i}$ sur $a_{i}$. En effet un isomorphisme de $M_{i}$ vers $L_{i}$ s'étend en un isomorphisme de $M_{i+1}$ vers $L_{i+1}$ envoyant $b_{i}$ sur $a_{i}$ : par induction $v M_{i}=v K$, donc $s_{i}$ reste Cauchy sur $M_{i}$; elle est sans limite dans $L_{i}$, donc dans $M_{i}$ par isomorphisme entre $M_{i}$ et $L_{i}$. Revenons à la conclusion du lemme. Il suffit de considérer $P(X)=\Sigma_{s} u_{s} X^{s}$ (où $X=\left(X_{1}, \ldots, X_{n}\right)$ et les $s$ sont des $n$-uplets d'entiers) avec des $u_{s} \in K$ de valuation $\geq 0$.

Puisque $P$ n'est pas identiquement nul, $\xi:=v\left(P\left(a_{1}, \ldots, a_{n}\right)\right)$ est dans $v K$, et pour tous $k_{1}, \ldots, k_{n} \in K$ tels que $v\left(k_{i}-a_{i}\right)>\xi$, on a $v\left(P\left(k_{1}, \ldots, k_{n}\right)\right)=$ $v\left(P\left(a_{1}, \ldots, a_{n}\right)\right)$.

Fait 22. Soit $(L, K, v)$ une paire $\aleph_{1}$-saturée de corps valués où $[L: K]$ est infini, et $B$ une boule de rayon non nul de $L$. Alors $\operatorname{tr}(K(B) ; K) \geq \aleph_{1}$; il en est de même si $B$ est une intersection dénombrable de telles boules.

\section{2. Élimination des quantificateurs}

Théorème 2. Les paires propres et denses de CVAC éliminent les quantificateurs dans le langage $\mathcal{L}_{\mathrm{div}}^{f}:=\mathcal{L}^{f} \cup \mathcal{L}_{\mathrm{div}}=\left\{0,1,+,-, \cdot \mid,\left(l_{n}\right)_{n \in \mathbb{N} \geq 2},\left(f_{n, i}\right)_{\substack{n \in \mathbb{N} \geq 2 \\ 1 \leq i \leq n}}\right\}$.

Les mêmes arguments que pour les paires de purs corps algébriquement clos montrent que ce théorème se déduit de l'élimination des quantificateurs dans le langage $\mathcal{L}_{v}^{c, f}:=\mathcal{L}^{c, f} \cup\left\{A_{v}\right\}$. Le preuve de cette élimination suit celle du cas des purs corps algébriquement clos. Le lemme suivant est le lemme 13 à l'identique avec $\mathcal{L}_{v}^{c, f}$ à la place de $\mathcal{L}^{c, f}$.

Lemme 23. Etant données une paire de corps algébriquement clos valués $\mathbb{M}$ et une $\mathcal{L}_{v}^{c, f}$-sous-structure $\mathbb{A} \subseteq \mathbb{M}$, la paire $\mathbb{A}^{a}:=\left(A^{a}, E_{A}^{a}\right)$ est une $\mathcal{L}_{v}^{c, f}$-sous-structure de $\mathbb{M}$ dont le type d'isomorphisme au-dessus de $A$ est imposé par celui de $\mathbb{A}$. 
Démonstration. Lemme 13 plus fait 17.

Le lemme suivant jouera le rôle qu'avait le lemme 11.

Lemme 24. Soient donnés une paire de corps valués algébriquement clos $\mathbb{M}$, une $\mathcal{L}_{v}^{c, f}$-sous-structure $\mathbb{A} \subseteq \mathbb{M}, A$ algébriquement clos, et un corps $B, E_{A} \subseteq B \subseteq E_{M}$, $B$ algébriquement clos et tel que $B$ est dense dans $A B$. Soit $\left(a_{i}\right)_{i<i_{0}}$ une base de transcendance de $A$ sur $E_{A}$ (donc de $A B$ sur $B$ ), et pour chaque $i<i_{0}$, $s_{i}=\left(b_{i, \alpha}\right)_{\alpha<\alpha_{0}}$ une suite de Cauchy de $B$ tendant vers $a_{i}\left(\alpha_{0}\right.$ est la cofinalité de $v B)$. Alors la paire $\mathbb{A} B:=(A B, B)$ est une $\mathcal{L}_{v}^{c, f}$-sous-structure de $\mathbb{M}$ dont le type d'isomorphisme au-dessus de $A$ ne dépend que des suites $s_{i}$ et du type d'isomorphisme du corps valué $B$ au-dessus de $E_{A}$.

Démonstration. Au vu du lemme 11, il ne reste qu'à vérifier que la valuation sur $A B$ est uniquement définie, ce qu'assure le lemme 21.

Un dernier lemme nous permettra de réaliser l'hypothèse du lemme précédent.

Lemme 25. Soient donnés une paire de corps valués $\mathbb{M}$, une $\mathcal{L}_{v}^{c, f}$-sous-structure $\mathbb{A} \subseteq \mathbb{M}$, et $x \in E_{M}$ vérifiant $v(x)>v A$. Alors la paire $\mathbb{A}(x):=\left(A(x), E_{A}(x), v\right)$ est une $\mathcal{L}_{v}^{c, f}$-sous-structure de $\mathbb{M}$ dont le type d'isomorphisme au-dessus de $\mathbb{A}$ est uniquement déterminé.

Démonstration. Par le lemme 11, $\mathbb{A}(x)$ est une $\mathcal{L}^{c, f}$-sous-structure de $\mathbb{M}$ et seule la détermination de la valuation sur $A(x)$ reste à vérifier. Or elle découle du fait 18.

Démonstration du théorème 2. Comme on l'a déjà fait remarquer, il suffit de montrer l'élimination des quantificateurs dans le langage $\mathcal{L}_{v}^{c, f}$. Nous utilisons de nouveau le critère de Shoenfield. On se donne donc $\mathbb{A}$ et $\mathbb{A}^{\prime}, \mathcal{L}_{v}^{c, f}$-sous-structures respectives des paires denses propres de cvac $\mathbb{M}$ et $\mathbb{M}^{\prime}$, où $\mathbb{M}$ est dénombrable, $\mathbb{M}^{\prime}$ $\aleph_{1}$-saturé, et où $\mathbb{A}$ et $\mathbb{A}^{\prime}$ sont isomorphes par un $\mathcal{L}_{v}^{c, f}$-isomorphisme $\sigma$. Il nous faut $\mathcal{L}_{v}^{c, f}$-plonger $\mathbb{M}$ dans $\mathbb{M}^{\prime}$ en prolongeant $\sigma$.

(1) On étend $\sigma$ de $\mathbb{A}^{a}$ vers $\mathbb{A}^{\prime a}$ grâce au lemme 23.

(2) On augmente $M$ et $A$ pour se ramener à une situation où, pour tout $x \in$ $A, v\left(x-E_{A}\right)$ est cofinal dans $v\left(x-E_{M}\right)$. Pour ce faire on considère $\mathbb{M}$ comme plongé dans une extension élémentaire $\aleph_{1}$-saturée $\mathbb{M}^{*}$, et on prend une base de transcendance $\left(a_{i} ; i<i_{0}\right)$ de $A$ sur $E_{A}$ (ainsi $\left.i_{0} \leq \omega\right)$, qui reste donc algébriquement libre sur $E_{M}$ et qu'on complète en une base de transcendance $\left(a_{i} ; i<j_{0}\right)$ de $M$ sur $E_{M}$ (donc $\left.j_{0} \leq i_{0}+\omega\right)$. On construit comme suit par induction sur l'entier $k$ des suites croissantes de corps $B_{k}$ et $M_{k}$ : on pose $B_{0}=E_{A}$ et $M_{0}=M$; supposons construits $B_{k-1}$ et $M_{k-1}$; pour chaque ordinal $i<j_{0}$, on prend $e_{k, i} \in E_{\mathbb{M}^{*}}$ vérifiant $v\left(e_{k, i}-a_{i}\right)>v\left(M_{k-1}\left(e_{k, 0}, \ldots, e_{k, i-1}\right)\right)$ et on définit $B_{k}:=B_{k-1}\left(e_{k, i} ; i<j_{0}\right)^{a}$ et $M_{k}:=\left(M_{k-1} B_{k}\right)^{a}$. Soit $B$ l'union des $B_{k}$ et $N$ celle des $M_{k}$. Par construction $a_{i}$ est limite de la suite $\left(e_{k, i}\right)_{k \in \omega}$; cette suite est Cauchy dans $N$, donc chaque $v\left(a_{i}-B\right)$ est cofinal dans $v\left(a_{i}-E_{N}\right)$. En particulier $E_{N}$ est dense dans $N$, ce qui permet de 
faire jouer à $\mathbb{N}$ le rôle de $\mathbb{M}$, et $B$ est dense dans $A B$, ce qui permet d'appliquer le lemme 25. On remplace $\mathbb{M}$ par $\mathbb{N}$ et $\mathbb{A}$ par $\mathbb{A} B$. Pour étendre $\sigma$ de $\mathbb{A}$ à $\mathbb{A} B$, il suffit par les lemmes 24 et 25, de trouver, pour chaque entier $k$ et chaque ordinal $i<j_{0}$, un élément $e_{k, i}^{\prime} \in E_{\mathbb{M}^{\prime}}$ vérifiant $v\left(e_{k, i}^{\prime}-\sigma\left(a_{i}\right)\right)>v\left(\sigma\left(M_{k-1}\left(e_{k, 0}, \ldots, e_{k, i-1}\right)\right)\right)$ et de poser $\sigma\left(e_{k, i}\right)=e_{k, i}^{\prime}$. De tels $e_{k, i}^{\prime}$ existent puisque la paire $\mathbb{M}^{\prime}$ est dense et $\aleph_{1}$-saturée. (3) Par le lemme $11, \mathbb{A} E_{N}$ est une $\mathcal{L}^{c, f}$-sous-structure de $\mathbb{N}$ et par les lemmes 25 et 21 son type de $\mathcal{L}_{v}^{c, f}$-isomorphisme au-dessus de $\mathbb{A} B$ est imposé par le type d'isomorphisme du corps valué $E_{N}$ au-dessus de $B$. Cela reste vrai pour sa clôture algébrique $C$. Par élimination des quantificateurs des CVAC dans le langage $\mathcal{L}_{v}$ et $\aleph_{1}$-saturation du corps valué $E_{M^{\prime}}$, il existe $D^{\prime} \subseteq E_{M^{\prime}}, D^{\prime}$ isomorphe à $E_{N}$ par un isomorphisme de corps valué qui est égal à $\sigma$ sur $B$. Ainsi $C$ et $\left(A^{\prime} D^{\prime}\right)^{a}=: C^{\prime}$ sont $\mathcal{L}_{v}^{c, f}$-isomorphes, appelons toujours $\sigma$ cet isomorphisme. Par $\aleph_{1}$-saturation de la paire $\mathbb{M}^{\prime}$, chaque suite $\left(\sigma\left(e_{i, n}\right)\right)_{n}, i_{0} \leq i<j_{0}$, admet une limite $a_{i}^{\prime}$ dans $E_{M^{\prime}}$. Par le lemme 21, $\sigma$ se prolonge en un $\mathcal{L}_{v^{-}}$-isomorphisme entre $N$ et $C^{\prime}\left(a_{i}^{\prime} ; i_{0} \leq i<j_{0}\right)^{a}$. Par le fait 22, on peut supposer les $a_{i}^{\prime}$ algébriquement indépendants au-dessus de $C^{\prime}$. L'isomorphisme entre $N$ et $C^{\prime}\left(a_{i}^{\prime} ; i_{0} \leq i<j_{0}\right)^{a}$ est alors un $\mathcal{L}_{v}^{c, f}$-isomorphisme.

Corollaire 26. La théorie des paires propres et denses de CVAC est modèlecomplète dans le langage $\mathcal{L}_{v}^{l d}:=\mathcal{L}^{l d} \cup \mathcal{L}_{v}=\left\{0,1,+,-, \cdot, A_{v},\left(l_{n}\right)_{n \in \mathbb{N} \geq 1}\right\}$.

Démonstration. Soient deux modèles $\mathbb{M}:=(M, K, v)$ et $\mathbb{M}^{*}:=\left(M^{*}, K^{*}, v\right), \mathbb{M}$ sous-structure de $\mathbb{M}^{*}$ au sens de $\mathcal{L}_{v}^{l d}$. Par modèle-complétude de la théorie des paires de purs corps algébriquement clos dans le langage $\mathcal{L}^{l d}$, l'inclusion $(M, K) \subseteq$ $\left(M^{*}, K^{*}\right)$ est élémentaire, donc $(M, K) \subseteq \subseteq_{\mathcal{L}^{c, f}}\left(M^{*}, K^{*}\right)$ d'où $\mathbb{M} \subseteq_{\mathcal{L}_{v}^{c, f}} \mathbb{M}^{*}$, et enfin $\mathbb{M} \preceq \mathbb{M}^{*}$.

Ce résultat d'élimination des quantificateurs permet de décrire dans [3] des expansions $C$-minimales et non définissablement complètes des CVAC.

Démonstration du corollaire 3, deuxième item. Par élimination des quantificateurs, des multiplets $x$ et $y$ de $M$ ont même type sur $C$ ssi, pour $D_{x}$ le plus petit corps contenant $C$ et $x$ et clos par les fonctions $f_{n, i},\left(D_{x}, E_{D_{x}}\right)$ et $\left(D_{y}, E_{D_{y}}\right)$ sont des paires de corps valués isomorphes au-dessus de $C$ par un automorphisme préservant $f$ et envoyant $x$ sur $y$. En particulier, si $x$ et $y$ sont dans $E_{M}$, ils ont même type sur $C^{a}$ dès qu'ils ont même type au sens du pur corps valué sur $E_{C^{a}}$. Il en résulte qu'un multiplet de $E_{M} \backslash C^{a}$ a une infinité de $\mathcal{L}_{v}^{c, f}$-conjugués sur $C^{a}$, donc un élément de $\left(C E_{M}\right)^{a} \backslash C^{a}$ aussi, et il en va de même pour un élément de $M \backslash\left(C E_{M}\right)^{a}$ sur $\left(C E_{M}\right)^{a}$. Donc $C^{a}$ reste la clôture algébrique de $K$ dans $M$ au sens de la paire de corps valués. Pour ce qui est de la clôture définissable, rappelons que, au sens des purs corps valués algébriquement clos, $\bar{C}:=\left(C^{p^{-\infty}}\right)^{h}=\left(C^{h}\right)^{p^{-\infty}}$ est la clôture définissable de $C$. En effet, pour un polynôme $P \in C[X]$ dans la situation de Hensel, c'est-à-dire unitaire, à coefficients de valuation $\geq 0$ et ayant une racine résiduelle simple, disons $\alpha$, le zéro de $P$ de reste $\alpha$, disons $a$, est définissable sur $C ; C^{h}$ étant obtenue à partir de $C$ par adjonctions successives de points dans la 
situation du a précédent, ses points sont définissables sur $K$; il en va de même de sa clôture parfaite $\bar{C}$. Réciproquement, puisque $\bar{C}$ est parfait, tout $x \in C^{a} \backslash \bar{C}$ admet un conjugué, au sens des corps, $y \neq x$; parce que $\bar{C}$ est henselien, $x$ et $y$ sont également conjugués au sens des corps valués. Ainsi $\bar{C}$ est la clôture définissable de $K$ dans $M$.

\section{References}

1. W. Baur, On the elementary theory of pairs of real closed fields II, J. Symbolic Logic 47 (1982) 669-679.

2. F. Delon, Indécidabilité de la théorie des paires immédiates de corps valués henseliens, J. Symbolic Logic 56 (1991) 1236-1242.

3. F. Delon, Corps $C$-minimaux, en l'honneur de François Lucas, Ann. Fac. Sci. Toulouse XXI (2012) 201-222.

4. I. Kaplansky, Maximal fields with valuation, Duke Math. J. 9 (1942) 303-321.

5. S. Lang, Introduction to Algebraic Geometry (Interscience, 1958).

6. G. Leloup, Théories complètes de paires de corps valués henseliens, J. Symbolic Logic 55 (1990) 323-339.

7. G. Leloup, Elimination des quantificateurs dans les paires de corps valués henseliens, J. Symbolic Logic 60 (1995) 548-562.

8. A. Macintyre, Classifying pairs of real closed fields, Ph.D. Dissertation, Stanford University, 1968.

9. B. Poizat, Paires de structures stables, J. Symbolic Logic 48 (1983) 239-249.

10. P. Ribenboim, Théorie des Valuations (Presses de l'Université de Montreal, 1964).

11. A. Robinson, Complete Theories (North-Holland, 1956).

12. A. Robinson, Solution of a problem of Tarski, Fund. Math. 47 (1959) 179-204.

13. J. R. Shoenfield, A theorem of quantifier elimination, Symp. Math. 5 (1971) 173-176. 